University of Nebraska - Lincoln

DigitalCommons@University of Nebraska - Lincoln

1980

\title{
The Cepheid Luminosity Scale
}

Edward G. Schmidt

University of Nebraska at Lincoln, eschmidt1@unl.edu

Follow this and additional works at: https://digitalcommons.unl.edu/physicsschmidt

Part of the Physics Commons

Schmidt, Edward G., "The Cepheid Luminosity Scale" (1980). Edward Schmidt Publications. 48.

https://digitalcommons.unl.edu/physicsschmidt/48

This Article is brought to you for free and open access by the Research Papers in Physics and Astronomy at DigitalCommons@University of Nebraska - Lincoln. It has been accepted for inclusion in Edward Schmidt Publications by an authorized administrator of DigitalCommons@University of Nebraska - Lincoln. 
THE CEPHEID LUMINOSITY SCALE

\author{
Edward G. Schmidt \\ Department of Physics and Astronomy \\ University of Nebraska \\ Lincoln, Nebraska, U.S.A.
}

The distance moduli of galactic clusters containing classical Cepheids are being redetermined using the four-color and $\mathrm{H} \beta$ photometric system. The results for four clusters are presented here and it is found that the distance moduli are smaller than previous values. Possible reasons for this discrepancy are discussed.

\title{
INTRODUCTION
}

The luminosities of Cepheids are important in the context of pulsation theory because they can be used together with effective temperatures to determine the pulsational masses of these stars. When this is done with currently accepted temperature and luminosity scales, the pulsational masses are in good agreement with the evolutionary masses. However, as discussed in the review papers by J.P. Cox and R.S. Stobie at this colloquium, the beat and bump masses are still too low when traditional homogeneous envelopes are used. Since the evolutionary masses are generally thought to be correct and since the pulsational masses agree with them, most efforts to alleviate this discrepancy have been directed at increasing the bump and beat masses. However, it should be noted that this viewpoint is dependent to a considerable extent on the luminosity scale which is used in obtaining the pulsational masses.

The luminosities of Cepheids in common use are based on those Cepheids which are members of galactic clusters. The luminosities of the Cepheids were determined from the distance moduli of the clusters which were obtained from the main-sequence fitting method. Although this is a well known method which has been used for many years, there are a number of factors which might affect the results obtained.

The Hyades main-sequence was used to set the zero point of the zero-age main sequence. Thus, changes in the distance of the Hyades will enter with full weight into the distance scale. It now appears that various methods of obtaining the distance of the Hyades are 
Püblished in SPACE SCIENCE REVIEWS 27 (1980), 4499-455. CopyrigCHAM4PS6 D. Reidel Publ./ Kluwer. Used by permission.

coming to be in good agreement (for discussions of this point, see de Vaucouleurs 1978 and Hansen 1980) and this is probably no longer an important source of uncertainty. However, when various clusters are fitted together to set up the ZAMS or to obtain the distances, it is necessary to assume that the main sequences are all identical. Van den Bergh (1977) has argued that the clusters with Cepheid members are likely to be less metal rich than the Hyades and that this will cause the distance moduli to be over-estimated by perhaps 0.15 mag. A further difficulty with main-sequence fitting arises due to contamination by field stars. Because the clusters with Cepheid members are all relatively distant, are generally substantially reddened and are close to the galactic plane, foreground field stars are easily confused with cluster members. For example, in NGC 129 it was found that half of the stars which appeared to be among the B stars of the cluster were in fact foreground stars. Finally, it should be noted that evolutionary effects can cause difficulties with the main-sequence fitting. For an example of the difficulties introduced by the combination of evolution and contaminatin, the reader is referred to the study of M25 by van den Bergh (1978). In the HR diagram which van den Bergh shows, it can be seen that among the brighter stars evolution is a problem while among the fainter stars there is enough contamination that the location of the main-sequence is not obvious. Clearly considerable judgement is required to obtain the distance modulus.

In view of the importance of the Cepheid luminosity scale and the potential difficulties with the main-sequence fitting method, it would be desirable to obtain independent distance estimates for the clusters in question. This paper reports on a program which is in progress with this purpose.

THE OBSERVATIONS

The B stars in clusters with Cepheid members are being observed in the Stromgren four-color and $H B$ system. The use of this system avoids the problems mentioned above in connection with the main-sequence fitting. The calibration of the system is independent of the Hyades and since B stars are being observed, the method is not affected by differential blanketing between various clusers. It is possible to discriminate against late type foreground stars which appear in the field of a reddened cluster and the effects of evolution on the $\mathrm{H} \beta$ index are small and well calibrated.

The four clusters for which the observations have been analyzed are listed in Table 1. The distance moduli in the fourth column are those given by Sandage and Tamann (1969) and increased by 0.2 magnitudes following Sandage and Tammann (1976). The calibration of the intermediate band photometry of Crawford (1978) was used with the present photometry to obtain the distance moduli in the next column. The seventh column gives the number of stars observed in each cluster 
TABLE 1

\begin{tabular}{|c|c|c|c|c|c|c|c|}
\hline \multirow[b]{2}{*}{ Cluster } & \multirow[b]{2}{*}{ Cepheid } & \multirow[b]{2}{*}{ Period } & \multicolumn{5}{|c|}{ True Distance Modulus } \\
\hline & & & S.\&T. & Present & $\begin{array}{c}\text { Standard } \\
\text { error } \\
\end{array}$ & $\begin{array}{c}\text { Stars } \\
\text { Observed } \\
\end{array}$ & $\begin{array}{c}\text { Stars } \\
\text { Rejected } \\
\end{array}$ \\
\hline \multirow[t]{3}{*}{ NGC 7790} & CE Cas a & 5.1 & 12.73 & 11.98 & \pm 0.15 & 16 & 5 \\
\hline & CE Cas b & 4.5 & & & & & \\
\hline & CF Cas & 4.9 & & & & & \\
\hline M25 & USgr & 6.7 & 9.18 & 8.44 & \pm 0.15 & 17 & 5 \\
\hline NGC 129 & DL Cas & 8.0 & 11.98 & 10.93 & \pm 0.19 & 22 & 11 \\
\hline NGC 6087 & $S$ Nor & 9.8 & 9.96 & 9.60 & \pm 0.09 & 16 & 5 \\
\hline
\end{tabular}

while the final column indicates how many were rejected as non-members. Data for two of the clusters, NGC 129 and NGC 6087, are already published (Schmidt 1980b, c) while the other two distance moduli are based on unpublished photometry.

It can be seen that the present distance moduli are smaller than the previous values by between .4 and $.8 \mathrm{mag}$. This result is displayed in terms of the pulsation masses in Figure 1. The pulsational masses (solid circles with error bars) in that diagram were obtained with the present distance moduli. Also shown are evolutionary masses (open circles) for the same stars. It can be seen that there is a significant mass discrepancy present. This would not be the case using Sandage and Tammann distances. Also shown for comparison are the masses of beat Cepheids (open triangles) and bump Cepheids (filled triangles) which were obtained from the literature (Fricke et al., 1972; King et al., 1975; Stobie, 1977). These masses were all calculated from homogeneous envelope models which had no magnetic fields.

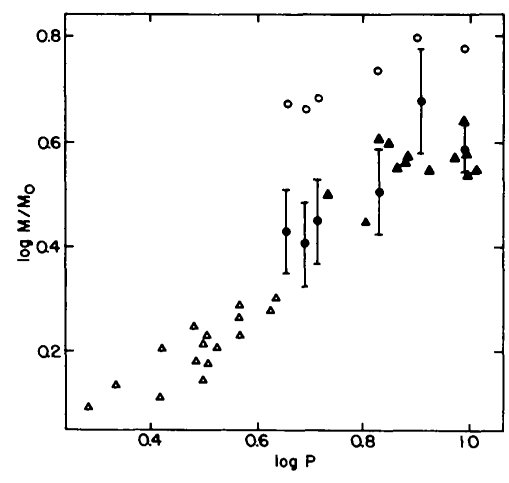

Figure 1. The masses of the Cepheids plotted against their periods. 


\section{POSSIBLE EXPLANATIONS FOR THE DISCREPANCY}

We mentioned above various factors which might affect the distance moduli obtained from the main-sequence fitting technique. Here we will consider the difficulties which might affect the distance moduli obtained from the intermediate-band photometry.

In Table 1 it can be seen that we have observed between 16 and 22 stars in each cluster. In most cases the number is limited by the number of $B$ stars in the cluster and the faintness of the stars. In any event, main-sequence fitting is generally done with a larger sample of stars. However, the results from the present photometry should not be affected adversely by this. This can be seen from the relatively small internal errors of the distance moduli. It is further shown in
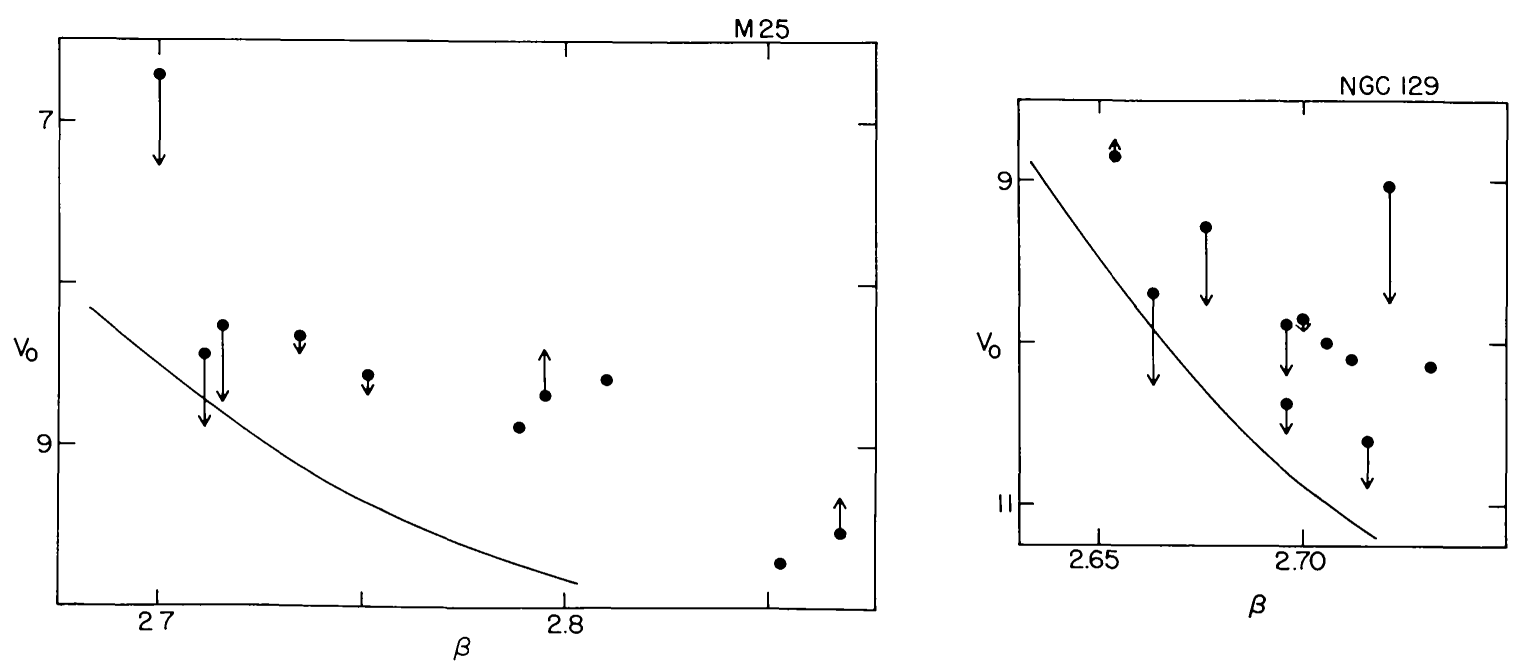

Figure 2. The $\mathrm{V}_{\mathrm{o}^{-\beta}}$ diagram for stars in two of the clusters.

Figure 2 where we have plotted the apparent magnitudes of the stars observed in two of the clusters against the $\mathrm{H} \beta$ indices. The arrows show the size of the evolutionary corrections for stars which required them. The solid curve is the relation between the absolute magnitude and the $\mathrm{H} \beta$ index adjusted to the Sandage and Tammann distance moduli. It can be seen that there is a clear discrepancy; in each cluster only one star scatters enough from the rest to reach the solid curve. Thus the discrepancy is not due to random errors or to too small a sample of stars.

To explain the present effect by systematic errors in the photometry would require errors in the $\mathrm{H} \beta$ indices of $0.03-0.04 \mathrm{mag}$. as can be seen in Figure 2. Such large errors are very unlikely. However, we can make a further check by comparing the present photometry for NGC 6087 and for stars in the field near 1 Car (Schmidt 1980a) with independent observations made by Eggen (1977, 1980). The systematic 
Published in SPACE SCIENCE REVIEWS 27 (1980), 449-455. Copyright $\odot 1980$ D. Reidel Publ./ Kluwer. Used by permission. differences between the present photometry and that of Eggen are less than $0.008 \mathrm{mag}$. for all indices. This indicates that the photometry is accurately on the standard system.

The absolute magnitude calibration of the intermediate band photometry is a further possible source of difficulty. The clusters studied here are similar to the Pleiades and the $\alpha$ Per clusters in age and the location of the turn-off point. Since these two clusters were used by Crawford as the basis for his calibration for B stars, it should be reliable in this region. A further check can be made by using the independent calibration of Eggen (1976). When the distances were redetermined using that calibration it was found that the distances decreased by between 0.0 and $0.3 \mathrm{mag}$. This reduces the discrepancy slightly but does not eliminate it.

In determining the mean distance moduli, some stars have been omitted as presumed field stars (as indicated in Table 1). The reasons for omitting individual stars included their position in the $\left[\mathrm{m}_{1}\right]-\left[\mathrm{c}_{1}\right]$ diagram, their location in the $\beta-c_{1}$ diagram or their having color excesses or distance moduli considerably different than the other cluster stars. In the case of NGC 7790, the inclusion of the rejected stars would only increase the mean modulus by $0.2 \mathrm{mag}$ while including the rejected stars for NGC 129 would decrease the modulus considerably. If we included all the stars in NGC 6087 and in M 25, the mean moduli of these two clusters would be brought into agreement with the Sandage and Tammann values. However, the stars in question are more distant than any of the other stars in the cluster by more than 1.5 magnitudes so their rejection is reasonable. Thus, it appears that the rejection of non-members is an important point but is not adequate to explain the distance scale discrepancy.

Finally, in view of the finding by Schmidt (1978) that some stars in NGC 129 and M 25 appear to be helium-weak stars, we should consider whether atmospheric helium abundance will affect the derived distance moduli. Calculated $\mathrm{H} \beta$ indices (Schmidt 1979) indicate that changing the number fraction of helium from 0.1 to 0.05 will change the index by less than $0.007 \mathrm{mag}$. This corresponds to an error in the absolute magnitude of less than $0.1 \mathrm{mag}$. We therefore conclude that the presence of helium weak stars is unlikely to cause the observed effect. In this we are assuming, of course, that the helium-weak phenomenon is an atmospheric effect and does not reflect the overall interior abundances of the stars.

\section{CONCLUSIONS}

The discrepancy between the luminosity scale for Cepheids derived from the intermediate band photometry and the presently accepted scale requires further investigation. We have discussed various factors which might affect the previous work and which might affect the present 
method but we are unable at this time to provide a definite explanation. However, since there is no obvious problem with the intermediate band photometry we should give serious consideration to the possibility that the Cepheid luminosity scale might require some revision.

\section{ACKNOWLEGEMENTS}

This work was supported by the National Science Foundation through Grant No. NSF AST77-17520 and by a Research Corporation Cottrell Research Grant. Many of the observations were obtained at Kitt Peak National Observatory and at Cerro Tololo Inter-American Observatory. The author is grateful to these observatories for the use of their facilities.

\section{REFERENCES}

Crawford, D.L.: 1978, Astron. J. 83, p. 48. de Vaucouleurs, G.: 1978, Astrophys. J. 223, p. 351. Eggen, 0.J.: 1976, P.A.S.P. 88, p. 241. Eggen, O.J.: 1977, Astrophys. J. Suppl. 34, p. 1. Eggen, 0.J.: 1980, Astrophys. J. 238, p. 919.

Fricke, K., Stobie, R.S., and Strittmatter, P.A.: 1972, Astrophys. J. 171, p. 593.

Hansen, R.B.: 1980, in "Star Clusters" IAU Symp. No. 85, ed. J.E. Hesser (Dordrecht: D. Reidel) p. 71.

King, D.S., Hansen, E.J., Ross, R.R., Cox, J.P.: 1975, Astrophys. J. 195, p. 467.

Sandage, A., and Tamman, G.A.: 1969, Astrophys. J. 157, p. 683.

Sandage, A., and Tammann, G.A.: 1976, Astrophys. J. 210, p. 7 .

Schmidt, E.G.: 1978, Astrophys. J. 219, p. 543.

Schmidt, E.G.: 1979, Astron. J. 84, p. 1739.

Schmidt, E.G.: 1980a, Astron. J. 85, p. 62.

Schmidt, E.G.: 1980b, Astron. J. 85, p. 158.

Schmidt, E.G.: 1980c, Astron. J. 85, p. 695.

Stobie, R.S.: 1977, M.N.R.A.S. 180, p. 631 .

van den Bergh, S.: 1977, Astrophys. J. 215, p. L103.

van den Bergh, S.: 1978, Astron. J. 83, p. 1174. 


\section{DISCUSSION}

A. COX: I am sitting here always trying to figure out how to get out of the box. What kind of $B$ stars are these?

SCHMIDT: They are B7 to B8 mainly. They are fairly cool.

A. COX: Then let me make my remark, which may not be so strong. There is an idea, not yet accepted, that the B stars have convection in which the turbulence decays very slowly. Cloutman and Whitaker have shown that you do extend the main sequence lifetime, but you also make it much more luminous because you have a lot more hydrogen and you burn it more vigorously. This could be a reason for making these stars a lot brighter than you thought.

SCHMIDT: Except that the calibration is all empirical, based on clusters very similar to these. If the effect is going on in these clusters, presumably it is going on in the calibration clusters. We ought to be getting the right answer.

A. COX: How do you get the ages of the clusters?

SCHMIDT: The ages don't come in. The Crawford calibration is based mainly on two clusters, the $\alpha$ Perseus cluster and the Pleiades. The $\alpha$ Perseus cluster is very similar to these. It even has $\alpha$ Persei which is almost a Cepheid. I think the calibration is pretty secure. It should be considerably better than for the earlier B stars.

FERNIE: B stars frequently have binary companions. Do you think this could have a systematic effect?

SCHMIDT: Again, the binaries have been left in the calibration and so, on the average, that ought not to affect anything. It is hard to imagine that these clusters are systematically different in binary content than the $\alpha$ Perseus cluster and the Pleiades but, obviously, that does have some effect. Crawford estimates that something like a couple tenths of a magnitude is the intrinsic scatter which includes binaries and other effects that are intrinsic.

SIMON: I would like to say that I think my colleague has done a very good job. I think the observers should have more to say. This is a very serious thing he is proposing.

PERCY: Can you compare this scale with the Barnes-Evans method scale?

SCHMIDT: My understanding is that their present scale is close to the Sandage and Tammann scale.

PERCY: Does this then introduce a discrepancy for which you have no explanation?

SCHMIDT: That is right.

BARNES: Our present scale makes the Cepheids brighter by about 0m03 \pm 0.2 from the Sandage-Tammann scale. It goes the other way. 